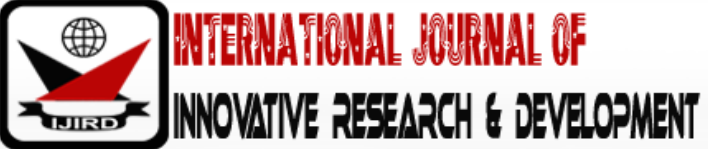

ISSN 2278 - 0211 (Online)

\section{Design and Implementation of Power Management System in a Complex Environment (PMSCE)}

\author{
Dr. Ebole Alpha. A \\ Senior Lecturer, Department of Computer Science, School of Technology, \\ Lagos State Polytechnic, Nigeria \\ Sarumi Jerry Abayomi \\ Senior Lecturer, Department of Computer Science, \\ School of Technology, Lagos State Polytechnic, Lagos State, Nigeria
}

\begin{abstract}
:
The introduction of Prepaid Energy Meter has led to power regulation were control of Voltages are made possible. But, for the actualization of energy in every home, there are needs for power conservation that allows equipment's in various homes to be synchronized to the assessment of the consumer in term of the user powering device(s) of interest. This Research work introduces a mobile phone used to control the activities of home appliances using short message services as a control method in a complex environment based on communication technology. The approach involves the connectivity of alternate current from the power supply, which is converted to direct current through the use of trigger transformer. The required voltage is disintegrated into $12 \mathrm{v}$ and $5 \mathrm{v}$ respectively. The microcontroller and the communication technology utilize the $5 \mathrm{v}$ while the relay and the buzzle make use of the $12 \mathrm{v}$. The software was programmed in such as to communicate with the microcontroller in other to regulate the mobile device that control the relay connected to different ports of home appliance. The result showed that a lot of energy was saved due to the control and regulation of devices of interest. In conclusion, it shown that high percentage of energy was served due to use ability to monitor home equipment of necessity, this also illustrate human and machine interaction in the communication medium and application of protocol in the current trend of internet of things (IOT). Moreover, it was observed that control of required voltage was also regulated by the transformer, whose function is to step up or stepdown voltage from the feeder.
\end{abstract}

Keywords: Prepaid energy meter, global system for mobile communications and microcontroller

\section{Introduction}

As the current trend of information technology, in term of Internet of Things (IOT) covering the global environment, power management system in a complex environment (PMSCE) was not left behind in the world of technological advancement. The development of super highway transmission and communication network in term of internet protocol prove way for the success story of human and machine interaction. They all try to solve those problems in this smart world, among these solutions; GSM has a well-known situation of mobile communication and has been a comprehensive information dissemination fashion in modern society. The prologue of GSM with wireless connectivity saw the light of Short Message Services (SMS) that used to send messages in the control of home appliances under a coded instruction on the microcontroller.

The human communication and machine interaction interface provide good technical possibility of remote monitoring and control to be the supremacy and convenience in other togreet way in Power Management System ina complex environment as people can monitor their home anytime, anywhere, in other to reduce the consumption of energy by their home equipment fuel to the use of prepaid energy meter. In this research work, presentation of wonderful power control management System as solution to energy conservation based on modern communication technology fostering the implementation and the concatenation of software and hardware as a means of solving National problems.

\subsection{Problem Statement}

The introduction of Prepaid Energy Meter has led to power regulation were control of Voltages were made possible. Most times, a lot of energy is wasted due to users powering equipment of no significant or at odd time, and inability of users powering off their equipment when there is power failure and in line to energy availability after a short way create an avenue for energy lose. 


\subsection{Objectives of the Study}

- The needs for power conservation that allows equipment's at various homes to be synchronized to the assessment of the consumer in term of the user powering device of interest.

- The control of home appliances using short message services method in a complex environment based on GSM (Global System for Mobile communications)

- The ability of the system to regulate voltage supply to each device

\section{Review of Related Literature}

The electronic makeup of humans' interaction and their environment can be illustrated in diverse conducts, which can be in form of simplex mode, half duplex and full duplex. The major communication to be applied is the full duplex because it allowed people to gain advantage in terms of performance and signal control of the environment. The power control management system has the capability to respond accordingly to the behavior of resident's financial position.

The concept of power management system in a complex environment has been urbanized since time in memorial. Recent definitions as stated by Satpathy, "as an intelligent home which is able to support the populaces in living independently and restfully with the help of technological transformation. Power management system, involves holistic interconnectivities of sub-system, component or interrelated parts to form a star network, where the switch/ router of many ports act as the master/ server and the home equipment act as the client/ slave and all communicate with each other in line with the user to create a collaborative galaxy" by Satpathy, L. Alam, M.R; Alauddin, M.A (2012). From the achieve, it has showed that power management system in a complex environment is automated to assist the users in different perspective: such as ambient acumen, remote home automation systems and above all to increase inhabitantswith more life springiness and make life enhancement as stated by Cook, D.J.; Das, S.K. on paper presentation on how smart are our environments (2007).

Power management system aims to establish an enhanced quality of living in line with prepaid meter by deploying fully-automated control of appliances of necessity by regulating and powering equipment of needs. It also allows end-users and appliances to have a handshake by transferring digital signals based on a standardized communication protocol. Power management system has a control communication device, which enables occupants to monitor prepaid token used and control home utilities from isolated environment as stated by Somayya, R.; Ramaswamy, R. Smart homes (2014). Le et al. (2014), and these pointed out that smart homes have the following five fundamental characteristics, such as automation, multi-functionality, adaptability, interactivity and efficiency.

However, smart griddoes not recognize power management system as stated by Erol-Kantarci, M.; Mouftah, H.T. (2010). The quest for Power management system to overcome Traditional Power Grids (TPG) give the rapidly rising electricity usage in private and public sector. Dynamism and productivity are the greatest necessities that power management system has a face with the smart grids. The applications of Smart technologies monitoring system were employed towork with home equipment energy usage, aggravated users to control home electricity through a half and full duplex transmission with home equipment.

It was understood, that power grid has been in use from time in memorial for the past 100 years, with a lot of power loses through generation, transmission, distribution and over deployment of power to various home-based equipment. To overcome these problems, the deployment of sensors, active computerization tools and bidirectional transmission are employed as stated by Javaid, N.; Khan, H.; Ullah, M.N.; Mahmood, A (2013).

There are needs for us to express the unit of power is watts in other to quantifier the capability of end user pattern of voltage consumption. One thousand watts is one kilowatt. If household equipment uses one kilowatt in one hour, it is cogitating as one unit of energy consumed for that particular home.

This can be express:

PULSE $=\underline{\text { PULSE RATE } * \text { WATT } * \text { TIME }}$

$\mathrm{PF} \quad=$ WATT

$$
1000 * 3600
$$

HOUR * PULSES

$\mathrm{UNITS}=\mathrm{PF} *$ TOTAL PULSE

$$
1000
$$

The important of energy meters is to measure voltage and currents by calculating its product in other to gives immediate solution to power consumption by home equipment. This power is unified over a specific period of time in other to give the energy utilized.

The necessity of the smart grid was its proficiency of refining enactment, dependability and customers' sensitivity towards the modification and advancement to the current trend of technology. The rapid development of ICT in providing solutions to smart metering as intelligent GSM prepaid energy meter have grown tackling the aforementioned limitations of existing power grids. The adaptation of traditional electricity grids into smart grids has led to greater productive and interactions among energy benefactors, operators and shareholders' by Mohsenian-Rad, A.H.; Leon-Garcia, A (2013). The deployment of smart prepaid meters, low-cost sensors and smart load devices, and the integration of ICTs in residential energy management programs has increases the efficiency of the energy by providing more automation, electrical loads, and a safer operation of electrical appliances, resulting in the quality of the energy delivery service and a higher overall user satisfaction Khan, I.; Mahmood, A.; Javaid, N.; Razzaq, S.; Khan, R.D.; Ilahi, M. (2015).

The functionality of the GSM in line with the network provided to automatically update the amount consumed and give current credit availability to follow the evolution of the balance between resource person and the consumer must be in real time. The end users can be trained to follow load-shifting programs that offer more effective way to manage the cost 
of their electricity bill as indicated in peak period and off-peak period by Graditi, G.; Ippolito, M.G.; Telaretti, E.; Zizzo, G. (2015).

\subsection{The New Role of the Users}

Inter-governmental Panel on Climate Change (2014) illustrate that adaptability to change on behavioral and attitude of users are another important factor in relation to the improvement of the energy performance of buildings. The data from Intergovernmental Panel on Climate Change (IPPC) showed that behavioral changes could affect energy savings in lighting by up to $70 \%$ IPPC.

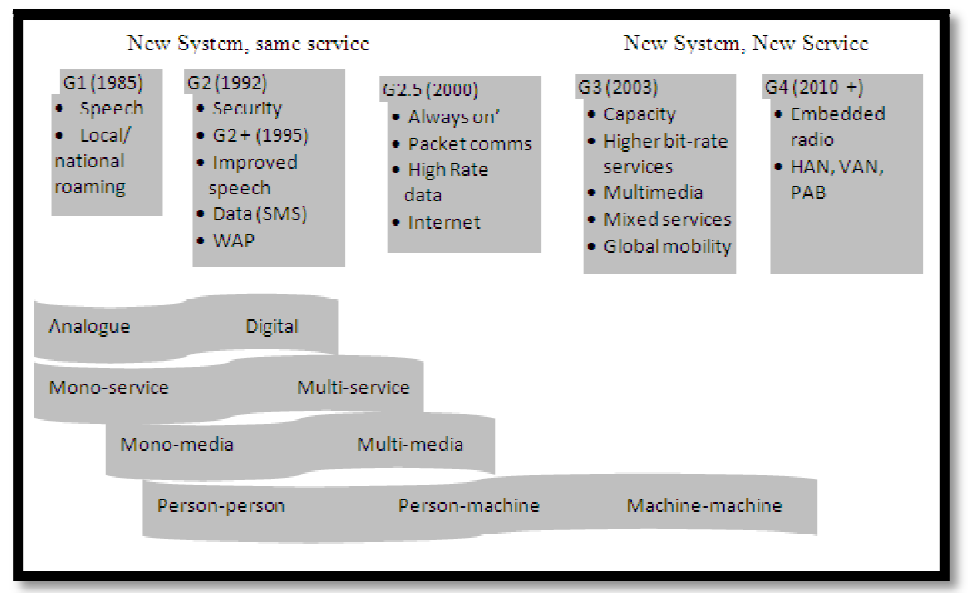

Figure 1: Changing Technologies and Service Models in Mobile Telephony Source: Rangrong Zhang, Xiaoping Zou, Wenhui Huang, Qimu-Surong, 2012

\begin{tabular}{|c|c|c|c|}
\hline Model & Relationship & Name & Characteristics \\
\hline 1 & $1: 1$ & Remote control & Half duplex transmission. \\
\hline 2 & $1: n$ & Smart appliance & Half duplex transmission remote system. \\
\hline 3 & n:1 & Smart system & Interconnectivity of the same subsystem \\
\hline 4 & n:n & Network of systems & Protocol advancement due to same networking \\
\hline 5 & N:N & $\begin{array}{c}\text { Intercommunicating } \\
\text { systems }\end{array}$ & $\begin{array}{c}\text { Different protocols advancement to different } \\
\text { network. }\end{array}$ \\
\hline
\end{tabular}

Table 1: Historical Trend of Information Technology (IT) Application

Notes: N =A Limited System or Set of Systems; N =A Large Set Of Systems

\section{Research Methodology}

The power management system comprises of transmission part, the cardinal control point and the entity to be controlled, as showed in the system block diagram. In this system, voltage from the smart prepaid energy meter is connected to the fuse box. From the fuse box, the voltage is link to the power management system that contain different port rages from 4ports, 6ports, 8ports (single phase), 16ports, 32ports, 64ports (Three phase) and 96ports, 128ports (Maximum Demand). The ports are connected to different device of end user equipment. Each port has voltage regulator that allow a specific load of dissimilar capacity to be controlled by the application of GSM Technology. The Microcontroller (PIC16F876A), is recognized as the control unification center. The relay componentis connected to home equipment help in triggering or switching circuit that define a particular port(s) to be active in providing services or describe the object to be controlled.

The Globe System for Mobile communication (GSM) has a module with a smart card incorporated into it forshort message transmission from the MCU (PIC16F876A)) to and fro the GSM network. It also receives message command from user's mobile and sends the corresponding command to the control point, which is the Microcontroller ('PIC16F876A). The Microcontroller investigate the command and demeanors the relay to open or close the anticipated channel or port, such to open or close the correlated home devices. The microcontroller authenticates the Globe System for Mobile communication module containing SIM 800L in other to send feedback message to the user terminal. 


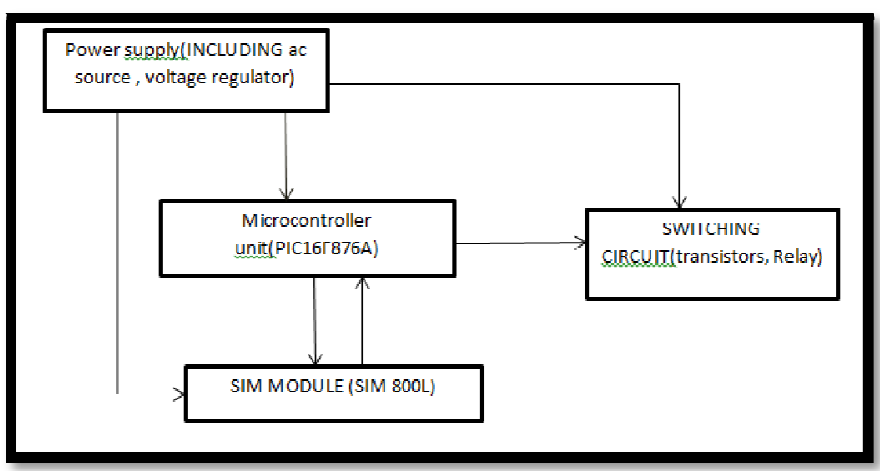

Figure 2: Block Diagram of Power Management System

Source: Author, 2019

\section{Attention Commands (At Commands)}

The Microcontroller interact with Globe System for Mobile communication (GSM)module through Attention Commands. The command act as interface standard between terminal equipment and terminal adaptor, as well as between data terminal equipment and data circuit terminal equipment, and connected to the GSM through hyper-terminal software which come with the OS.

\subsection{Hardware Structure}

Hardware component comprises of two parts: board communications and a GSM module. Component on the boardis the relay module located at the upper side and bellow is a microcontroller board on it. The GSM module is connected to the center board through a pair of UART ports of PIC16F876A controller. GSM Module exchanges data with user terminal through the GSM network provided why the microprocessor (PIC16F876A) transferals data with TC351 module through transmitter port and receiver port. The segment of the relay controls Input / Output ports and each port is directly proportional to each links at home usage. The diagram below illustrates the schematic diagram of the GSM module and MCU.

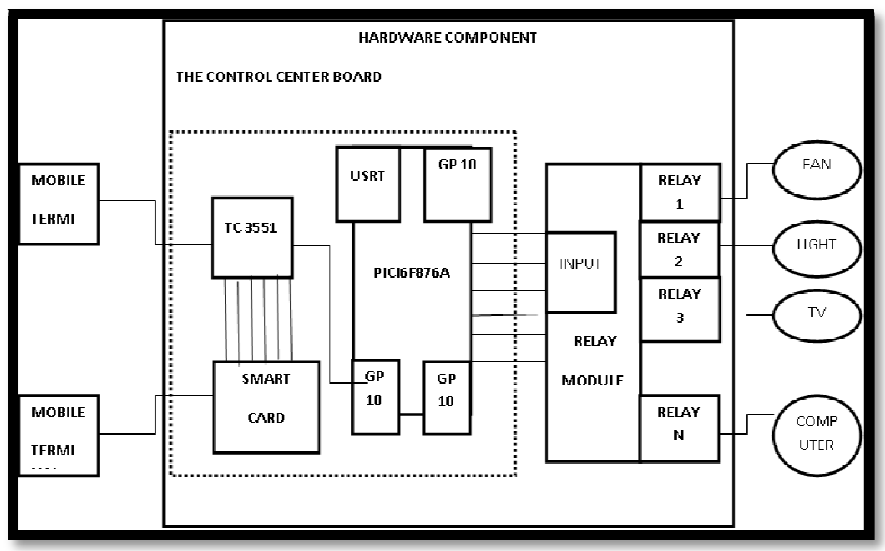

Figure 3: The Interface between Globe System for Mobile Communication and the Microcontroller Source: Author, 2019

Below is the simulation of the main board of the system using Altium Designer

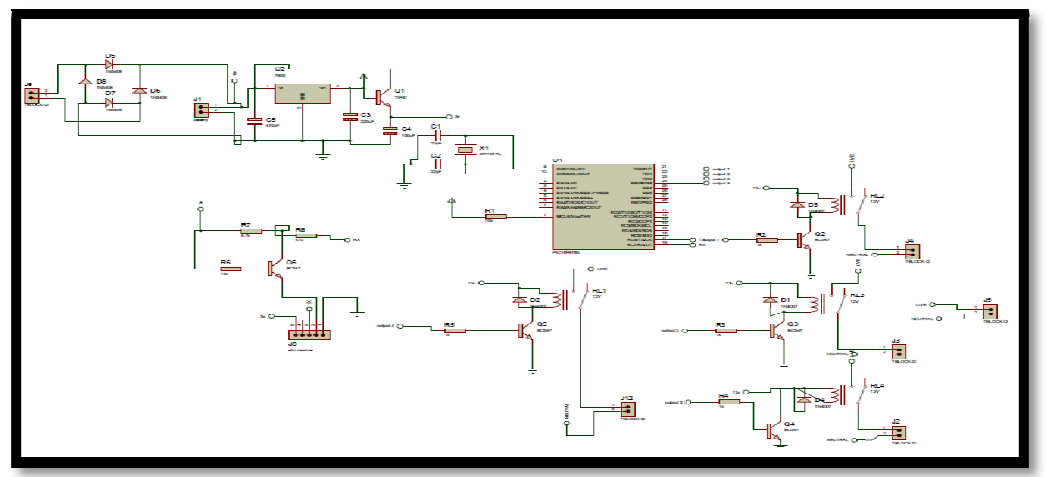

Figure 4: Simulation of Power Management System

Source: Author, 2019) 
Microcontroller is linked to the GSM module, the GSM module employed was the TC351 of SIEMENS because of its efficiency and ability to break bearers. The TC351 module can work at specific frequency and able to transmit sound, data, message and fax. It has a data transmission period of $8 \mathrm{~V}-30 \mathrm{~V}$ and $1.5 \mathrm{mps}$ for lower devices and work voltage is $5.6 \mathrm{~V}-30 \mathrm{~V}$, and $12 \mathrm{mps}$ for high devices.

The MCU, was necessary due to the following features: ultra-low work voltage watchdog, double-speed, lower EMI, timer, ISP, IAP, ultra-small-scale power consumption, 63Kbyte FLASH, 1280-Byte RAM, and uses the universal asynchronous receiver and transmitter.

\subsection{Software Framework}

System software is divided into two parts: SMS for sending \& receiving part and SMS parsing \& execution part. The function of sending \& receiving is completed by GSM module which is mainly responsible for receiving remote control commands and returning the status of the system +to remote control terminals; the control section is completed by the MCU, which will parse the received GSM commands so as to control the corresponding peripherals and communication is achieved through serial port connected to MUC.

\subsection{Software Flowchart}

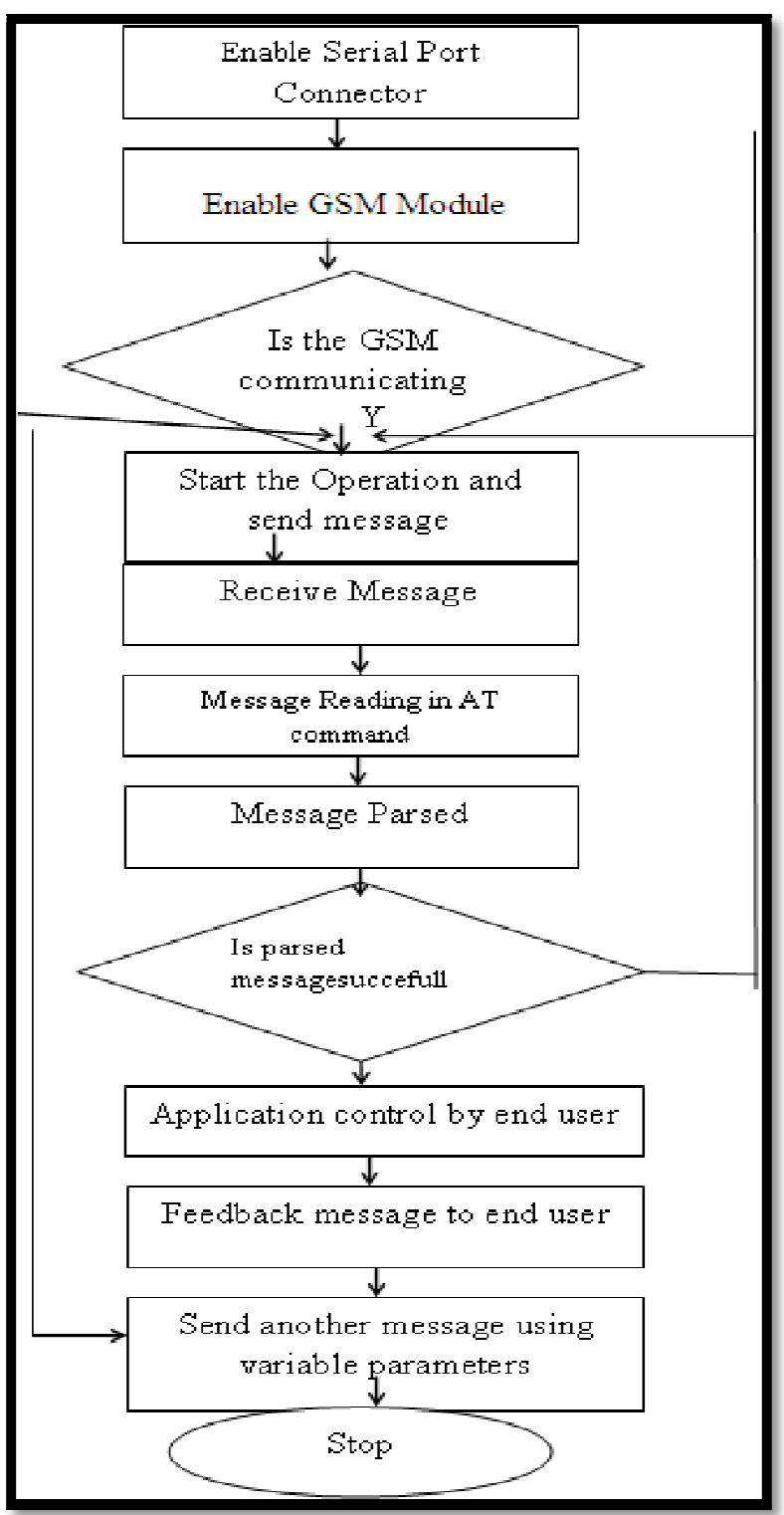

Figure 5: MUC Communication with the GSM

\section{Software Tests and Results Discussion}

Mobile phone application demonstrates the power of authoritarian text information transmitted tenuously in other to regulate the work condition of power management system. Base on the specified port, if message is sent to "port 1 " by the user send to the GSM module containing the SIM card number, the illumination on that particular port or channel is emitted. Feedback message from the system to the GSM indicating that an operation is carried out. If we use other commands like "port 2", "port 3" ... and so on other devices will also be opened. In this way, we realize the meaning of total regulation of home devices where GSM network is covered. It is necessary to note that, the buzzle control and regulate data in the mobile phone and radiate alarm at a particular interval indicating the position of the recharge card. 


\section{Conclusions}

Introduction of power management system requires full integration of devices to prove the technology of IOT and its capabilities in the realization of consumer satisfaction and regulation of device(s) of interest. The processes and interprocesses to interaction remnants at the core of power management system. However, the makeup of power management system involves the use of communications medium and protocol. Therefore, there are needs for the development of sophisticated approach to understanding the processes by which commercialization and socialization of power management system products and services in terms of operations most be established.

\section{Contribution to knowledge}

This system makes remote control available based on GSM Technology in controlling device(s) of necessity, by so doing reducing the concept of over utilization and wastage of voltage of no necessity in a complex environment. It also makes sense in industry control and other fields by demonstrating the current tendency of intelligent system.

\section{Future Research}

Sensor technology most be provided in other to support multimedia applications in term of Audio and video display, also parameters like temperature and humidity for controlling the sensitive home appliance as mobile phone tends to be like a mini computer.

\section{References}

i. Win Adiyansyah Indra, Fatimah Bt Morad, Norfadzlia Binti Mohd Yusof and Siti Asma Che Aziz, "GSM-Based Smart Energy Meter with Arduino Uno", International Journal of Applied Engineering Research, Vol. 13, No. 6, pp. 39483953, 2018.

ii. M. Patel Umang and M. Modi Mitul, "A Review on Smart Meter System", International Journal of Innovative Research in Electrical, Electronics, Instrumentation and Control Engineering, Vol. 3, No. 12, December 2015.

iii. M. S. Vidyashree, "GSM Based Smart Energy Meter to Implement Billing System and To Control Electricity Theft", International Journal of Current Engineering and Scientific Research (IJCESR), Vol. 4, No. 1, 2017.

iv. Samarth Pandit, Sneha Mandhre, Meghana Nichal, "Smart Energy Meter using internet of Things (IoT)", VJERVishwakarma Journal of Engineering Research, Vol. 1, No. 2, pp. 222-229, June 2017.

v. Win Hlaing, Somchai Thepphaeng, Varunyou Nontaboot, Natthanan Tangsunantham, Tanayoot Sangsuwan and Chaiyod Pira, "Implementation of Wi-Fi-Based Single Phase Smart. Meter for Internet of Things (IoT)", 5th International Electrical Engineering Congress, Pattaya, Thailand, pp. 8-10, March 2017.

vi. N. Darshan Iyer and Dr. K. A. Radhakrishna Rao, "IoT Based Electricity Energy Meter Reading, Theft Detection and Disconnection using PLC modem and Power optimization", International Journal of Advanced Research in Electrical, Electronics and Instrumentation Engineering, Vol. 4, No. 7, pp. 6482-6491, July 2015.

vii. S. Imran and Dr. K. Prahlada, "IOT Based Electricity Energy Meter Reading Through Internet", International Journal of Technical Innovation in Modern Engineering \& Science (IJTIMES), Vol.3, No. 11, November-2017.

viii. Libiao Qiao, Xiaojun Liu and Baochen Jiang, "Design andImplementation of the Smart Meter in Vehicle-to-Grid", IEEE 2012.

ix. Alan S. Fung, Farah Mohammadi, and Kaamran Raahemifar and Ramyar Rashed Mohassel, "A Survey on Advanced Metering Infrastructure and its Application in Smart Grids ", IEEE CCECE 2014.

x. Amit Jain and Mohnish Bagree, "A prepaid meter using mobile communication", International Journal of Engineering, Science and Technology Vol. 3, No. 3, pp. 160-166, 2011.

xi. Mr.P. Gokula Krishnan and Ms.K. Arunachaleswari, "Distributed Generation Grid Infrastructure using Smart Meters Modeled with Renewable Energy Sources and Power Trading "ISSN: 2277-9655 Impact Factor: 1.852, December, 2013.

xii. C. Gao and M. A. Redfern, "A Review of Voltage Control in Smart Grid and Smart Metering Technologies on Distribution Networks", 46th International Universities' Power Engineering Conference, ISBN 978-3-8007-34023,8th September 2011. 